\title{
I Lepidotteri Ropaloceri della Riserva Statale di Popolamento Animale "Lago di Burano" (Capalbio, Grosseto)
}

\begin{abstract}
Riassunto: Negli anni 2007-2009 è stata effettuata una ricerca sui Lepidotteri Ropaloceri della Riserva Statale di Popolamento Animale "Lago di Burano" (Capalbio, GR). Le specie accertate sono 50 tra le quali otto (Thymelicus acteon, Gegenes nostrodamus, Zerynthia cassandra, Lycaena thersamon, Charaxes jasius, Libythea celtis, Hipparchia fagi, Hipparchia statilinus) di interesse conservazionistico. Le entità più diffuse sono Pieris brassicae, Colias crocea, Polyommatus icarus, Lasiommata megera, Pieris rapae, Gonepteryx cleopatra, Maniola jurtina, Coenonympha pamphilus, Papilio machaon, Limenitis reducta e Kanetisa circe, specie in grado di colonizzare diverse situazioni ambientali, quelle sporadiche Thymelicus sylvestris, Ochlodes venatus, Zerynthia cassandra, Euchloe ausonia, Favonius quercus, Satyrium ilicis, Cacyreus marshalli, Celastrina argiolus, Polyommatus thersites, Inachis io e Polygonia c-album, che non trovano nella riserva ambienti idonei alla loro sopravvivenza. Le principali minacce per i Ropaloceri di Burano sono rappresentate dalla gestione della vegetazione operata per fini agricoli e dalla regimazione delle acque. Per ridurre l'impatto di questa attività si suggeriscono: il mantenimento di una fascia di terreno incolto (ampia almeno $20 \mathrm{~m}$ ) intorno al lago e di una striscia di vegetazione (larga tra $2 \mathrm{e} 4 \mathrm{~m}$ ) lungo i canali retrostanti; la conservazione di siepi e arbusti; l'effettuazione degli interventi di sfalcio della vegetazione erbacea tra dicembre e gennaio.
\end{abstract}

Abstract: Butterflies of the "Lago di Burano" State Reserve (Capalbio, Grosseto)

During 2007-2009 we surveyed rhopalocerans in the Riserva Statale di Popolamento Animale "Lago di Burano" (southern Tuscany). We found fifty species, some of which are widespread and common (Pieris brassicae, Colias crocea, Polyommatus icarus, Lasiommata megera, Pieris rapae, Gonepteryx cleopatra, Maniola jurtina, Coenonympha pamphilus, Papilio machaon, Limenitis reducta and Kanetisa circe), but others, such as Thymelicus acteon, Gegenes nostrodamus, Zerynthia cassandra, Lycaena thersamon, Charaxes jasius, Libythea celtis, Hipparchia fagi and Hipparchia statilinus, are threatened. The main threats to rhopalocerans in the reserve are vegetation and water control in agriculture. To reduce the impact of agriculture it is suggested: i) to maintain a strip of uncultivated land at least $20 \mathrm{~m}$ wide around the lake and a belt of vegetation 2-4 m wide along channels and ditches; ii) to conserve hedges and shrubs; iii) to cut herbaceous plants in December or January.

Key words: Lepidoptera, Rhopalocera, Tuscany, Riserva Statale di Popolamento Animale "Lago di Burano", checklist, distribution, conservation.

\section{INTRODUZIONE}

Il Lago di Burano costituisce uno dei biotopi lacustri costieri di maggiore interesse dell'Italia centro-meridionale per le peculiarità floristiche, vegetazionali e faunistiche, dovute alla permanenza di habitat propri delle zone umide, oggi scomparsi in gran parte d'Italia per la bonifica, la messa a coltura e l'urbanizzazione. Per queste ragioni sul suo territorio sono stati istituiti la prima oasi del WWF in Italia, una Riserva Statale di Popolamento Animale, una Zona di Protezione Speciale (ZPS 133 "Lago di Burano") e due Siti di Importanza Comunitaria (pSIC 131 "Lago di Burano" e pSIC 132 "Duna del Lago di Burano"). È stato, inoltre, dichiarato "Zona umida di importanza internazionale" ai sensi della Convenzione di Ramsar ed è stato incluso tra le Important Bird Areas (IBA) dal BirdLife International (Bardi et al., 2002).

La presente ricerca, frutto di un accordo tra il WWF Italia e il Dipartimento di Scienze Ambientali
dell'Università di Siena, è stata promossa al fine di acquisire informazioni sui Lepidotteri Ropaloceri, uno dei gruppi faunistici meno noti nel comprensorio, per il quale sino ad oggi erano note solo tre specie, Papilio machaon Linné, 1758, Zerynthia cassandra (Geyer, 1828) e Danaus chrysippus (Linné, 1758) (Giusti et al., 1993; Cianchi \& Sestieri, 2008).

\section{Materiali e Metodi}

AREA DI STUDIO. L'area esaminata coincide con i limiti della Riserva Statale di Popolamento Animale "Lago di Burano". Questa riserva, istituita nel 1980, è situata nel comune di Capalbio (Grosseto), tra il Promontorio di Ansedonia e la foce del Fosso Chiarore. Comprende il bacino lacustre, uno stagno costiero salmastro, e parte dei terreni circostanti per una superficie complessiva di circa 410 ettari (Bardi et $a l ., 2002)$. Il substrato è formato in prevalenza da sedimenti torbosi e alluvionali di origine lacustre e pa- 
lustre alternati a sabbie oloceniche e pleistoceniche (Bardi et al., 2002). Il clima è di tipo mediterraneo, con temperatura media annua di $15,6^{\circ} \mathrm{C}$. Le precipitazioni sono scarse e concentrate per lo più nei mesi di gennaio e febbraio $(654,4 \mathrm{~mm}$ annui presso la stazione pluviometrica di Monte Alzato) (Chelazzi et al., 1990; Barazzuoli et al., 1993). Nel lago la vegetazione dominante è costituita da ampie fasce di canneto a canna palustre (Phragmites australis), da prati umidi e da salicornieti a salicornia fruticosa ( $\mathrm{Ar}$ throcnemum fruticosum), salicornia europea (Salicornia europea) e suaeda marittima (Suaeda maritima). Le aree retrostanti il lago sono estesamente coltivate mentre i cordoni di duna che separano il bacino lacustre dal mare sono caratterizzati da vegetazione erbacea pioniera con il ravastrello marittimo (Cakile maritima) e lo sparto pungente (Ammophila littoralis), da una macchia mediterranea a ginepro coccolone (Juniperus oxycedrus var. macrocarpa) e ginepro fenicio (Juniperus phoenicea) e da un lembo residuo di foresta sempreverde a leccio (Quercus ilex) e sughera (Quercus suber), con qualche roverella (Quercus pubescens) e farnetto (Quercus frainetto) (Angiolini et al., 2002).

CAMPIONAMENTO E IDENTIFICAZIONE. Il territorio della riserva è stato suddiviso in unità di rilevamento, coincidenti con il reticolato chilometrico UTM ED 1950 - Fuso 32 (base cartografica impiegata: Carta Topografica Regionale 1:250.000 Ed. $1^{\mathrm{a}}, 1980$ ). In questo modo la riserva è risultata ripartita in nove quadrati: PN9496-9498, 9596-9597, 9696-9697, 9796-9797 (Fig. 1); altri due quadrati, PN9397 e PN9598, non sono stati presi in considerazione poichè includono porzioni molto marginali di territorio dell'area protetta.

I campionamenti sono stati effettuati negli anni 2007-2009: il primo anno dal 18.01 al 18.10, il secondo dal 29.02 al 06.10 , il terzo dal 12.05 al 13.08. In media è stata eseguita un'uscita al mese, per un totale di 25 uscite e 175 ore di osservazione. Per reperire il maggior numero di specie sono state visitate tutte le tipologie ambientali esistenti nelle unità di rilevamento, privilegiando per le osservazioni le ore centrali della giornata (tra le 10:00 e le 16:00), periodo durante il quale i lepidotteri risultano più attivi e, quindi, più facili da rilevare (Pollard \& Yates, 1994). Tutte le farfalle sono state determinate sul campo direttamente a vista, nel caso di specie in-

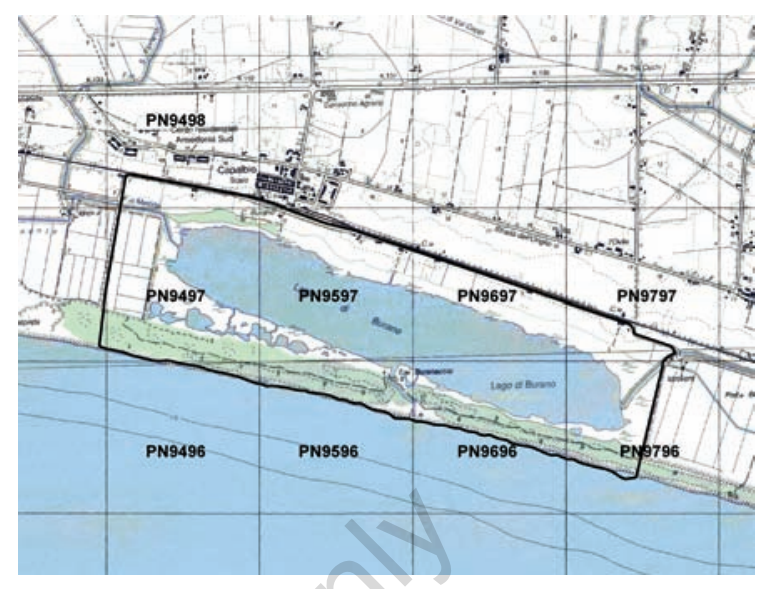

Fig. 1. Area di studio con le 9 unità di rilevamento UTM $1 \times 1 \mathrm{~km}$ campionate.

confondibili, o dopo averle catturate mediante retini entomologici, nel caso di specie non riconoscibili senza essere manipolate.

TASSONOMIA E NOMENCLATURA. La tassonomia e la nomenclatura seguono Balletto et al. (2007) con esclusione del genere Zerynthia per il quale è stato seguito Dapporto (2010).

\section{Risultati e Discussione}

CATAlogo delle SPecie. Nel territorio della riserva sono state accertate 50 specie di ropaloceri (Tab. 1). Delle tre specie segnalate in passato l'unica non ritrovata è Danaus chrysippus (Linné, 1758), entità a corotipo afrotropicale-indiano-mediterraneo in progressiva espansione Italia (Balletto et al., 2007, 2009). È probabile che la sua presenza nella riserva sia sporadica in quanto dovuta a individui in dispersione come d'altronde avvenuto in altre località costiere del grossetano (Fiume Bruna presso Castiglione della Pescaia; Parco Naturale della Maremma; Talamone; dintorni di Capalbio e dintorni del Lago di San Floriano) (Terzani, 1998; L. Favilli \& S. Piazzini, dati personali inediti).

Rispetto a quella del vicino Parco Naturale Regionale della Maremma, per il quale sono note 68 specie (Nappini \& Dapporto, 2009), la ropalocerofauna di Burano risulta decisamente più povera. Probabilmente ciò è dovuto alla ridotta estensione dei 
Tab. 1. Checklist e copertura dei Lepidotteri Ropaloceri della Riserva Statale di Popolamento Animale "Lago di Burano".

\begin{tabular}{|c|c|c|}
\hline Specie & Unità di rilevamento coperte & $\%$ di presenza \\
\hline \multicolumn{3}{|l|}{ Famiglia Hesperiidae } \\
\hline Pyrgus armoricanus (Oberthür, 1910) & 2 & $22,2 \%$ \\
\hline Pyrgus malvoides (Elwes \& Edwards, 1897) & 1 & $11,1 \%$ \\
\hline Carcharodus alceae (Esper, 1780) & 3 & $33,3 \%$ \\
\hline Thymelicus acteon (Rottemburg, 1775) & 2 & $22,2 \%$ \\
\hline Thymelicus sylvestris (Poda, 1761) & 2 & $22,2 \%$ \\
\hline Thymelicus lineola (Ochsenheimer, 1808) & 2 & $22,2 \%$ \\
\hline Ochlodes venatus (Bremer \& Grey, 1853) & 2 & $22,2 \%$ \\
\hline Gegenes nostrodamus (Fabricius, 1793) & 3 & $33,3 \%$ \\
\hline \multicolumn{3}{|l|}{ Famiglia Papilionidae } \\
\hline Papilio machaon Linné, 1758 & 5 & $55,6 \%$ \\
\hline Iphiclides podalirius (Linné, 1758) & 1 & $11,1 \%$ \\
\hline Zerynthia cassandra (Geyer, 1828) & 2 & $22,2 \%$ \\
\hline \multicolumn{3}{|l|}{ Famiglia Pieride } \\
\hline Pieris brassicae (Linné, 1758) & 7 & $77,8 \%$ \\
\hline Pieris edusa (Fabricius, 1777) & 4 & $44,4 \%$ \\
\hline Pieris mannii (Mayer, 1851) & 3 & $33,3 \%$ \\
\hline Pieris napi (Linné, 1758) & 2 & $22,2 \%$ \\
\hline Pieris rapae (Linné, 1758) & 6 & $66,7 \%$ \\
\hline Euchloe ausonia (Hübner, 1804) & 1 & $11,1 \%$ \\
\hline Anthocharis cardamines (Linné, 1758) & 3 & $33,3 \%$ \\
\hline Colias alfacariensis Ribbe, 1905 & 3 & $33,3 \%$ \\
\hline Colias crocea (Geoffroy, 1785) & 7 & $77,8 \%$ \\
\hline Gonepteryx cleopatra (Linné, 1767) & 6 & $66,7 \%$ \\
\hline Gonepteryx rhamni (Linné, 1758) & 3 & $33,3 \%$ \\
\hline \multicolumn{3}{|l|}{ Famiglia Lycaenidae } \\
\hline Lycaena phlaeas (Linné, 1761) & 4 & $44,4 \%$ \\
\hline Lycaena thersamon (Esper, 1784) & 3 & $33,3 \%$ \\
\hline Favonius quercus (Linné, 1758) & 1 & $11,1 \%$ \\
\hline Satyrium ilicis (Esper, 1779) & 2 & $22,2 \%$ \\
\hline Cacyreus marshalli Butler, 1898 & 1 & $11,1 \%$ \\
\hline Leptotes pirithous (Linné, 1767) & 4 & $44,4 \%$ \\
\hline Lampides boeticus (Linné, 1767) & 3 & $33,3 \%$ \\
\hline Celastrina argiolus (Linné, 1758) & 1 & $11,1 \%$ \\
\hline Aricia agestis (Denis \& Schiffermüller, 1775) & 4 & $44,4 \%$ \\
\hline Polyommatus icarus (Rottemburg, 1775) & 7 & $77,8 \%$ \\
\hline Polyommatus thersites (Cantener, 1834) & 1 & $11,1 \%$ \\
\hline \multicolumn{3}{|l|}{ Famiglia Nymphalidae } \\
\hline Inachis io (Linné, 1758) & 1 & $11,1 \%$ \\
\hline Vanessa atalanta (Linné, 1758) & 4 & $44,4 \%$ \\
\hline Vanessa cardui (Linné, 1758) & 3 & $33,3 \%$ \\
\hline Polygonia c-album (Linné, 1758) & 1 & $11,1 \%$ \\
\hline Issoria lathonia (Linné, 1758) & 2 & $22,2 \%$ \\
\hline Melitaea didyma (Esper, 1778) & 2 & $22,2 \%$ \\
\hline Melitaea phoebe (Denis \& Schiffermüller, 1775) & 4 & $44,4 \%$ \\
\hline Charaxes jasius (Linné, 1767) & 4 & $44,4 \%$ \\
\hline Limenitis reducta Staudinger, 1901 & 5 & $55,6 \%$ \\
\hline \multicolumn{3}{|l|}{ Famiglia Libytheidae } \\
\hline Libythea celtis (Laicharting, 1782) & 1 & $11,1 \%$ \\
\hline \multicolumn{3}{|l|}{ Famiglia Satyridae } \\
\hline Kanetisa circe (Fabricius, 1775) & 5 & $55,6 \%$ \\
\hline Hipparchia fagi (Scopoli, 1763) & 4 & $44,4 \%$ \\
\hline Hipparchia statilinus (Hufnagel, 1766) & 1 & $11,1 \%$ \\
\hline Maniola jurtina (Linné, 1758) & 6 & $66,7 \%$ \\
\hline Coenonympha pamphilus (Linné, 1758) & 6 & $66,7 \%$ \\
\hline Pararge aegeria (Linné, 1758) & 3 & $33,3 \%$ \\
\hline Lasiommata megera (Linné, 1767) & 7 & $77,8 \%$ \\
\hline
\end{tabular}


Tab. 2. Specie di importanza conservazionistica e loro inclusione nelle principali liste rosse come specie minacciate o prossime alla minaccia (categorie secondo IUCN, 2001).

\begin{tabular}{lcccc}
\hline Specie & IUCN (2011) & $\begin{array}{c}\text { Van Swaay et al. } \\
(2010)\end{array}$ & $\begin{array}{c}\text { Balletto et al. } \\
(2007)\end{array}$ & $\begin{array}{c}\text { Fabiano et al. } \\
(2001)\end{array}$ \\
\hline $\begin{array}{l}\text { Thymelicus acteon (Rottemburg, 1775) } \\
\text { Gegenes nostrodamus (Fabricius, 1793) }\end{array}$ & NT & EN & VU \\
$\begin{array}{l}\text { Zerynthia cassandra (Geyer, 1828) } \\
\text { Lycaena thersamon (Esper, 1784) }\end{array}$ & & & VU \\
$\begin{array}{l}\text { Charaxes jasius (Linné, 1767) } \\
\text { Libythea celtis (Laicharting, 1782) }\end{array}$ & & & VU \\
Hipparchia fagi (Scopoli, 1763) & NT & NT & NT \\
Hipparchia statilinus (Hufnagel, 1766) & & NT & & \\
\hline
\end{tabular}

Legenda: EN: Endangered; NT: Near Threatened; VU: Vulnerable.

prati-pascoli e della macchia sempreverde, habitat ben rappresentati, invece, nel Parco della Maremma e in grado di ospitare una ricca ropalocerofauna con entità di interesse quali Melanargia arge (Sulzer, 1776) e Coenonympha elbana Staudinger, 1901 la cui assenza a Burano non è altrimenti spiegabile, dal momento che entrambe sono note per località immediatamente limitrofe (L. Favilli \& S. Piazzini, dati personali inediti). Interessante rimane, comunque, la presenza di otto specie di importanza conservazionistica a livello globale, europeo e regionale, minacciate o prossime ad esserlo in seguito a distruzione e frammentazione dell'habitat causata dalle attività antropiche (sviluppo dell'agricoltura intensiva, uso di pesticidi ed erbicidi, abbandono delle praterie seminaturali, distruzione dei boschi e delle siepi, incendi, urbanizzazione) (Prola \& Prola, 1990; Van Swaay \& Warren, 1999; Fabiano et al., 2001; Balletto et al., 2007; Van Swaay et al., 2010; Van Swaay et al. in IUCN, 2011). Si tratta di Thymelicus acteon, Gegenes nostrodamus, Zerynthia cassandra, Lycaena thersamon, Charaxes jasius, Libythea celtis, Hipparchia fagi e H. statilinus (Tab. 2).

Distribuzione nella Riserva. Pieris brassicae, Colias crocea, Polyommatus icarus e Lasiommata megera risultano le specie più diffuse, avendo i valori più alti di copertura $(77,8 \%)$ seguite da Pieris rapae, Gonepteryx cleopatra, Maniola jurtina e Coenonympha pamphilus $(66,7 \%$ di copertura) (Tab. 1). Si tratta, infatti, di entità euriecie, in grado di stabilirsi perfino in ambienti con forte presenza antropica (Balletto et al., 2007). G. cleopatra, in particolare, è ritenuta specie caratterizzante i consorzi a macchia mediterranea degli ambienti costieri (Balletto et al., 1989; Audisio, 2002), ed è, infatti, molto comune lungo tutto il litorale della Toscana meridionale (L. Favilli \& S. Piazzini, dati personali inediti). Abbastanza diffuse sono Papilio machaon, Limenitis reducta e Kanetisa circe (55,6\% di copertura), anche queste entità ampiamente distribuite in Italia (Balletto et al., 2007) e decisamente comuni in Toscana meridionale (L. Favilli \& S. Piazzini, dati personali inediti) (Tab. 1).

Da poco diffuse a sporadiche risultano tutte le altre specie (copertura tra il 44,4\% e l'11,1\%). La ridotta diffusione di entità nemorali come Favonius quercus, Satyrium ilicis, Celastrina argiolus e $\mathrm{Pa}$ rarge aegeria è da mettere in relazione con l'assenza di estese formazioni forestali. Per le stesse ragioni specie tipicamente legate alle situazioni ecotonali tra il bosco e le aree aperte come Ochlodes venatus, Anthocaris cardamines, Inachis io e Polygonia c-album mostrano una distribuzione notevolmente ridotta. Apparentemente sorprendente è la bassa copertura di Charaxes jasius, entità tipicamente legata ai boschi mediterranei e caratteristica degli ambienti costieri del versante tirrenico della penisola italiana (Balletto et al., 2007), legata probabilmente alla rarità del corbezzolo (Arbutus unedo), pianta ospite della larva, nella riserva.

Il fatto, invece, che quasi tutte le zone aperte 
dell'area protetta siano intensamente coltivate, spiega bene la scarsissima diffusione di entità proprie di formazioni erbacee caratterizzate da buoni valori di naturalità come Carcharodus alceae, Thymelicus sylvestris, Euchloe ausonia, Colias alfacariensis e Polyommatus thersites. La sporadica diffusione di Zerynthia cassandra e Pieris mannii potrebbe essere correlabile con il fatto che si tratta di specie localizzate e mai particolarmente comuni in Toscana e in Italia centrale (Favilli et al., 2004; Balletto et al., 2007; L. Favilli \& S. Piazzini, dati personali inediti). Considerazioni a parte merita Cacyreus marshalli. Si tratta di un licenide originario del Sud Africa introdotto accidentalmente in Europa a partire dalla fine degli anni ' 80 in seguito all'importazione di gerani coltivati infestati con uova o larve dal Sud Africa (Favilli \& Manganelli, 2006; Balletto et al., 2007). Al di fuori dell'areale originario C. marshalli appare legato agli ambienti antropici: frequenta, infatti, quasi esclusivamente terrazze e giardini dove sono presenti gerani, piante ospiti del bruco. In alcuni paesi europei, come la Spagna, è diventato un vero flagello per le piante coltivate (Sarto i Monteys, 1992) e recenti osservazioni sembrano dimostrare che sia anche in grado di attaccare i gerani spontanei (Quacchia et al., 2008). In un prossimo futuro C. marshalli potrebbe espandersi nella Riserva Statale di Popolamento Animale "Lago di Burano", favorito dalle temperature medie annue abbastanza elevate e dall'ampio utilizzo che viene fatto dei gerani come piante ornamentali in tutto il comprensorio.

\section{CONCLUSIONI}

La ropalocerofauna della Riserva Statale di Popolamento Animale "Lago di Burano" non è particolarmente ricca ed è costituita per lo più da entità ampiamente diffuse in Italia e in Toscana che non presentano particolari problemi di conservazione. Otto specie, al contrario, risultano rare, poco comuni, minacciate o prossime a esserlo a livello europeo, nazionale e regionale e per questo rivestono un particolare interesse. Tra esse meritano di essere ricordate G. nostrodamus e L. thersamon, per le quali l'area del Lago di Burano rappresenta uno dei pochi siti toscani dove sono presenti (Balletto et al., 2007; Favilli et al., 2006; Favilli et al., in stampa).

Sebbene la gestione a fini conservazionistici operata da oltre quaranta anni sul territorio della riserva garantisca un certo grado di tutela alla fauna, sono attivi alcuni fattori di rischio che minacciano lo stato di conservazione di molte specie di Ropaloceri. Il più significativo è rappresentato dalla ripulitura delle sponde dei canali operata per fini agricoli e per la regolazione del flusso delle acque, che limita fortemente la sopravvivenza della popolazione di $Z$. cassandra. Questa farfalla, infatti, si sviluppa a spese delle specie di Aristolochia (Balletto et al., 2007) che crescono lungo le sponde dei canali. La loro ripulitura, con la conseguente distruzione della vegetazione ripariale, può portare alla scomparsa della specie. I frequenti sfalci della vegetazione erbacea operati nel periodo di attività di larve e adulti dei lepidotteri, insieme alla rimozione di siepi e arbusti effettuati per fini agricoli, risultano altamente deleteri in quanto distruggono i siti di alimentazione, rifugio e svernamento di molte specie. Le più danneggiate sono entità poco vagili e meno adattabili come Carcharodus alceae, Lycaena thersamon e Polyommatus thersites (Balletto \& Kudrna, 1985), per le quali gli incolti della riserva rappresentano siti di vitale importanza.

Per mantenere in buono stato di conservazione le popolazioni di ropaloceri della riserva si ritiene, quindi, necessario realizzare alcuni interventi finalizzati alla gestione e alla tutela degli habitat e alla pianificazione delle attività agricole. In particolare gli interventi dovrebbero prevedere: il mantenimento di una fascia di terreno incolto lungo il perimetro del lago, ampia almeno $20 \mathrm{~m}$, e di una striscia di vegetazione lungo i canali retrostanti il lago, larga tra 2 e $4 \mathrm{~m}$, nelle quali lasciar sviluppare la vegetazione spontanea necessaria per l'alimentazione di larve e adulti; il mantenimento di siepi e arbusti, anch'essi importanti come siti di rifugio e alimentazione; l'effettuazione degli interventi di sfalcio della vegetazione erbacea tra dicembre e gennaio quando i bruchi non sono attivi, in modo tale da evitare la loro distruzione. Questi interventi hanno costi e tempi di realizzazione estremamente ridotti e consentono una buona tutela dei Ropaloceri (Balletto, 2005; Boriani et al., 2005; Tellini Florenzano et al., 2010).

\section{RINGRAZIAMENTI}

La ricerca è stata effettuata, in parte, con contributo del WWF Oasi (Prot. no. W059/07). Un ringraziamento particolare a Pamela Rustici e a Martina Stolzi per aver collaborato alla raccolta dei dati. 


\section{BIBLIOGRAFIA}

Angiolini C., Riccucci C., De Dominicis V., 2002 - La flora vascolare della Riserva Naturale Lago di Burano (Toscana meridionale, Grosseto). Webbia, 57: 115-152.

Audisio P., 2002 - Litorali sabbiosi e organismi animali. In: Audisio P., Muscio G., Pignatti S. \& Solari M., Dune e spiaggie sabbiose. Ambienti fra terra e mare. Quaderni Habitat, 4. Dune e spiagge sabbiose. Ministero dell'Ambiente e della Tutela del Territorio, Museo Friulano di Storia Naturale, Udine: 63-117.

Balletto E., 2005 - Lepidotteri In: C. Blasi, L. Boitani, S. La Posta, F. Manes \& M. Marchetti (a cura di), Stato della biodiversità in Italia. Contributo alla strategia nazionale per la biodiversità. Ministero dell'Ambiente e della Tutela del Territorio, Direzione per la Protezione della Natura, Società botanica italiana. Palombi Editore, Roma: 256-261.

Balletto E., Barbero F., Casacci L.P., Cerrato C., Patricelli D. \& Monelli S., 2009 - L'impatto dei cambiamenti climatici sulle farfalle italiane. Studi Trentini di Scienze Naturali, 86: 111-114.

Balletto E., Bonelli S. \& CAssulo L., 2007 - Insecta Lepidoptera Papilionoidea (Rhopalocera). In: Ruffo S., Stoch F. (eds.), Checklist and distribution of the italian fauna. Ministero dell' Ambiente e della tutela del territorio, Direzione per la protezione della natura. CD-ROM.

BALletto E. \& KudRnA O., 1985 - Some aspects of the conservation of butterflies in Italy, with recommendations for a future strategy (Lepidoptera Hesperiidae \& Papilionoidea). Bollettino della Società Entomologica Italiana, 117: $39-59$.

Balletto E., Toso G. \& Lattes A., 1989 - Studi sulle comunità di Lepidotteri Ropaloceri del litorale tirrenico. Bollettino dei Musei e degli Istituti Biologici dell’Università di Genova, 53: 141-186.

Barazzuoli P., Guasparri G. \& Salleolini M., 1993 - Il clima. In: Giusti F. (a cura di), La storia naturale della Toscana meridionale. Amilcare Pizzi Editore, Milano: 141-171.

Bardi A., Iacobini M., Persia G., Petretti F., Lenzi M., Caspari A., Donzelli F., Violante D. \& Piazzi A., 2002 - Piano di gestione della Riserva Naturale Lago di Burano. Temi s.r.l., Ministero dell’Ambiente e Tutela del Territorio, WWF Italia Onlus, inedito

Boriani L., Burgio G., Mariani M. \& Genghini M., 2005 - Faunistic study on butterflies collected in Northern Italy rural landscape. Bullettin of Insectology, 58: 49-56.

Chelazzi L., Colombini I., Bertin G., Cianfanelli A., Fallaci M., Lucarelli E. \& Mascagni A., 1990 - Gli artropodi del tombolo antistante la Laguna di Burano (GR): ambiente, microclima e primi dati sul popolamento animale. Redia, 73: 307-345.

DAPPORTO L., 2010 - Speciation in mediterranean refugia and post-glacial expansion of Zerynthia polyxena (Lepidoptera, Papilionidae). Journal of Zoological Systematics and Evolutionary Research, 48: 229-237.

Fabiano F., Vignali G. \& Dapporto L., 2001 - Lepidotteri. In: Sforzi A. \& Bartolozzi L. (a cura di), Libro rosso degli Insetti della Toscana. ARSIA Regione Toscana, Firenze: 293-343.

FAVILli L. \& MANGANelli G., 2006 - Life history of Cacyreus marshalli, a South African species recently introduced into Italy (Lepidoptera Lycaenidae). Bollettino della Società Entomologica Italiana, 138: 51-62.

Favilli L., Piazzini S. \& Manganelli G., 2004 - Nuovi dati sulla distribuzione in Toscana meridionale di alcuni lepidotteri diurni (Papilionoidea, Hesperoidea) rari o poco noti. Atti della Società Toscana di Scienze Naturali Residente in Pisa Memorie Serie B, 110: 25-29.

Favilli L., Piazzini S. \& Manganelli G., 2006 - Segnalazioni faunistiche italiane (N. 442-452). Lycaena thersamon (Esper, 1784) (Lepidoptera Lycaenidae). Bollettino della Società Entomologica Italiana, 138: 79.

Favilli L., Piazzini S., Tellini Florenzano G., Perroud B. \&, Manganelli G., in stampa - Nuovi dati sulla distribuzione in Toscana di alcuni lepidotteri diurni rari o poco noti (Hesperoidea, Papilionoidea). Atti della Società Toscana di Scienze Naturali Residente in Pisa Memorie Serie B, 117.

IUCN, 2001 - IUCN Red List categories and criteria. Version 3.1. http://www.iucnredlist.org/documents/redlist_cats_crit_en.pdf (ultimo accesso: 19.10.2011).

IUCN, 2011 - The IUCN Red List of Threatened species. http://www.iucnredlist.org/ (ultimo accesso: 28.09.2011).

NApPini S. \& DApporto L., 2009 - I macrolepidotteri del Parco Regionale della Maremma (Toscana) (Lepidoptera). Memorie della Società Entomologica Italiana, 88: 177-195.

Pollard E. \& Yates T., 1993 - Monitoring Butterflies for Ecology and Conservation. Chapman \& Hall, London.

Prola G. \& Prola C., 1990 - Libro rosso delle farfalle italiane. WWF Quaderni, 13: 71 pp., 16 Tavv. 
Quacchia A., Ferracini C., Monelli S, Balletto E. \& Alma A., 2008 - Can the geranium bronze, Cacyreus marshalli, become a threat for european biodiversity? Biodiversity and Conservation, 17: 1429-1437.

Tellini Florenzano G., Acciaioli A., Piazzini S., Londi G. \& Malvezzi R., 2010 - 6. Linee guida per la gestione delle praterie. In: Acciaioli A., Londi G. \& Tellini Florenzano G. (a cura di), La gestione e il recupero delle praterie dell'Appennino settentrionale. Il pascolamento come strumento di tutela e salvaguardia della biodiversità. Collana Manuali Arsia, Regione Toscana, Firenze: 43-48.

TERZANI F., 1982 - Segnalazioni Faunistiche Italiane. 17. Coenonympha elbana Staudinger (Lepidoptera Satyridae). Bollettino della Società Entomologica Italiana, 114: 117.

TERZANI F., 1998 - Sulla presenza di Danaus chrysippus (Linnaeus, 1758) in Toscana (Lepidoptera Danaidae). Atti del Museo di Storia Naturale della Maremma, 17: 193-194.

VAN SWAAY, C.A.M., WARREN R.S., 1999 - Red Data Book of european butterflies (Rhopalocera). Council of Europe Nature Environment Series, 99: 1-264.

Van Swaay C., Cuttelod A., Collins S., Maes D., Munguira M.L., Sasic M., Settele J., Verovnik R., Verstrael T., WarREN M., Wiemers M. \& Wynhoff I., 2010 - European Red List of Butterflies. Pubblication Office of the European Union, Luxembourg, $60 \mathrm{pp}$.

Indirizzo degli autori:

S. Piazzini, E. Spadini, F. Cianchi, L. Favilli \& G. Manganelli, Dipartimento di Scienze Ambientali, Via Mattioli 4, I53100 Siena, Italia. favilli@unisi.it 